\title{
Estrés en empleados de un ingenio azucarero veracruzano
}

\author{
Stress in employees of a sugar mill in Veracruz \\ Pressão sobre funcionários de uma usina de açúcar Veracruzano
}

María Esther Barradas Alarcón

Universidad Veracruzana, México

ebarradas@uv.mx

Javier López González

Universidad Veracruzana, México

jalopez@uv.mx

Sonia Báez Lagunés

Instituto tecnológico de Veracruz, México

soniabaezl@hotmail.com

Elvitz de los Ángeles. Gutiérrez Vázquez

Universidad Veracruzana, México

elvgutierrez@uv.mx

\section{Resumen}

Esta investigación tuvo como objetivo identificar el nivel de estrés, en de diferentes áreas en un Ingenio de Veracruz. Orientación metodológica es cuantitativa y el nivel de estudio descriptivo. Instrumento utilizado fue el test Perfil de Estrés Kenneth M. Nowak. La población fue 52 empleados. Resultados, con una distribución del 100\% para cada área, en nivel alto de estrés se tuvo; un $28 \%$ en jefes, un $20 \%$ en empleados de campo, un $9 \%$ en empleados del área técnica-mecánica, y no presentaron estrés los empleados del área administrativa. Conclusión, Aunque si bien es cierto que el porcentaje de nivel alto de estrés, es menor, se considera necesario capacitar en el manejo de técnicas de afrontamiento para todos los empleados.

Palabras claves: Nivel de estrés. 


\section{Abstract}

This research aimed to identify the level of stress, in different areas in a Veracruz Mill. Methodological orientation is quantitative and descriptive level of study. Instrument used was the Stress Profile test Kenneth M. Nowak. The population was 52 employees. Results, with a distribution of $100 \%$ for each area, in high level of stress had; $28 \%$ in bosses, $20 \%$ in field employees, $9 \%$ in technical-mechanical employees, and employees in the administrative area did not present stress. Conclusion Although it is true that the percentage of high level of stress is lower, it is considered necessary to train in the handling of coping techniques for all employees.

Key words: stress level.

\section{Resumo}

Esta pesquisa teve como objetivo identificar o nível de estresse em diferentes áreas em Ingenio Veracruz. abordagem metodológica é o nível de estudo quantitativo e descritivo. Instrumento de teste utilizado foi o perfil de tensões Kenneth M. Nowak. A população foi de 52 funcionários. Resultados, com a distribuição de 100\% para cada área, alto nível de estresse tinha; $28 \%$ cabeças, $20 \%$ em funcionários de campo, $9 \%$ na área funcionários técnico-mecânico, e não mostrou empregados estresse da área administrativa. Conclusão Embora seja verdade que a percentagem de alto nível de estresse é menor, considera-se necessário para treinar na manipulação de técnicas de enfrentamento para todos os funcionários.

Palavras-chave: nível de estresse.

Fecha recepción: Enero $2016 \quad$ Fecha aceptación: Junio 2016 


\section{Introducción}

Vivir en esta época sin estrés, es una utopía, ya que este es considerado como un mal irremediable. Independientemente del contexto donde se trabaje, el estrés se encuentra presente.

De acuerdo a la Organización Internacional del Trabajo (OIT) menciona que México es uno de los países con mayor índice de trabajadores que presentan estrés laboral en un 75\%, de ahí China presenta un $73 \%$ y los Estados Unidos presentan un 59\%

Según un informe de la Universidad Sussex (Rodríguez, 2001), de Inglaterra, México es uno de los países con mayores niveles de estrés en el mundo, debido a que presenta los principales factores que provocan estrés, tales como pobreza y cambios constantes de situación. Y las consecuencias de padecerlo no solo se ven reflejadas en el trabajador sino también en la empresa y por lo tanto el país. Razón por la que se realiza la siguiente investigación con el objetivo de conocer el nivel de estrés que presentan los trabajadores del Ingenio la Gloria.

\section{Estrés}

El concepto de "estrés" fue utilizado por primera vez por Hans Selye en 1936 para definir el estrés en términos biológicos como "una respuesta inespecífica del cuerpo a cualquier solicitud de cambio". El estrés puede referirse a una condición negativa o una condición positiva que responda a un factor estresante y que pueda tener un impacto en la salud mental o física y en el bienestar de una persona.

El estrés es la respuesta de adaptación con la mediación de características individuales y/o procesos psicológicos, y que es consecuencia de cualquier acción,

de acuerdo a la Organización Internacional del Trabajo (OIT. 2015:s/p), el estrés laboral es una "enfermedad peligrosa para las economías industrializadas y en vías de desarrollo; perjudicando a la producción, al afectar a la salud física y mental de los trabajadores”

Para la OIT, el estrés es la respuesta física y emocional a un daño causado por un desequilibrio entre las exigencias percibidas y los recursos y capacidades percibidos de un individuo para hacer frente a esas exigencias. 
Partiendo el paradigma que la salud está reconocida como una combinación de factores biológicos, psicológicos (pensamientos, emociones y comportamientos), y sociales (socioeconómicos, socio ambientales, y culturales).

En síntesis entonces las consecuencias del estrés laboral son mutiles nuevamente la organización internacional explicó que este padecimiento "no sólo impacta la salud de los ciudadanos, sino directamente la economía de los países, que representa pérdidas de entre 0.5 y $0.3 \%$ del Producto Interno Bruto de cada nación. Por su parte el Instituto Nacional de Estadística y Geografía (INEGI) menciono, que el Producto Interno Bruto (PIB) de México cerró durante el 2014 con un incremento de $2.1 \%$, por lo que este padecimiento de salud representa pérdidas millonarias para toda la República mexicana. Algunas de las causas son el volumen y ritmo de trabajo, las tareas aburridas o monótonas, empleos inestables, elevado nivel de responsabilidad, exposición a tareas insalubres o peligrosas, falta de apoyo, acoso por parte de sus compañeros o superiores, utilización inadecuada de la posiciones de poder, falta de reconocimiento, condiciones ambientales del lugar de trabajo, combinación de cualquiera de los anteriores factores. En si el estrés es generado no solo por una causa si de la presencia de varias de ellas, lo que favorece que los niveles de estrés se incremente y afecte al empleado como a la empresa.

\section{Metodología}

\section{Descripción del Método.}

Tipo de investigación. El estudio es de tipo descriptivo que de acuerdo a Hernández, Fernández y Baptista (2014), pretende establecer las características, los niveles de asociación, las formas de manifestación o los índices de ocurrencia del problema de investigación.

Método. Se realizó a través de una metodología cuantitativa; que según Hernández, Fernández y Baptista (2014) es el método que utiliza la recolección y el análisis de datos para contestar preguntas de investigación, confiando en la medición numérica, el conteo y frecuentemente en el uso de la estadística para establecer con exactitud patrones de comportamiento en una población.

Definición de variable.- El estrés es la respuesta de adaptación con la mediación de características individuales y/o procesos psicológicos, y que es consecuencia de cualquier 
acción, situación o acontecimiento externo que inflige demandas físicas y/o psicológicas a las personas. (Lazarus, R. 1981).

Instrumento.- el test "Perfil de Estrés" de Kenneth M. Nowack, el cual proporciona una amplia evaluación del estrés y del riesgo para la salud incluyendo factores que demuestran relación entre estrés y enfermedad. Consta de 123 reactivos, conformado por siete áreas; estrés, hábitos de salud, red de apoyo social, conducta tipo A, fuerza cognitiva, estilo de afrontamiento y bienestar psicológico; dividido en 15 sub-escalas relacionadas con el estrés y el riesgo de enfermedad. Las propiedades psicométricas: confiabilidad por mitades de 0,89 y 0,91 en poblaciones de diferentes razas y nivel educativo. La homogeneidad de las escalas con confiabilidad test-retest muestran un rango de 0,51 a 0,92 alpha de Cronbach y los análisis factoriales las reportan con baja a moderada relación ( 0,41 a 0,75 eigen).

Objetivo de Investigación.- identificar el nivel de estrés, y técnicas de afrontamientos de empleados de diferentes departamentos de un Ingenio azucarero del Estado de Veracruz.

Población.- realizó 66 trabajadores que ocupan diversos puestos tipos (45) de todas las áreas administrativas, operativas y gerenciales. Siendo en total cuarenta y cinco hombres y veinte mujeres que se aplicaron dichos instrumentos. Tabla 1 
Tabla 1.- Datos sociodemográficos de la población estudiada.

\begin{tabular}{cccc}
\hline CATEGORÍA & CARACTERISTICA & N & $\%$ \\
\hline Sexo & Hombre & 47 & 71.2 \\
& Mujer & 19 & 28.7 \\
Edad & & & \\
& 22 a 30 años de edad & 4 & 6.0 \\
& 31 a 40 años de edad & 15 & 15,1 \\
& 41 a 50 años de edad & 19 & 16.6 \\
& 51 a 61 años de edad & 28 & 25.7 \\
\hline
\end{tabular}

Fuente: Propia

\section{ANÁLISIS DE LOS RESULTADOS}

En cuanto a la presencia de estrés se tiene la siguiente figura 1

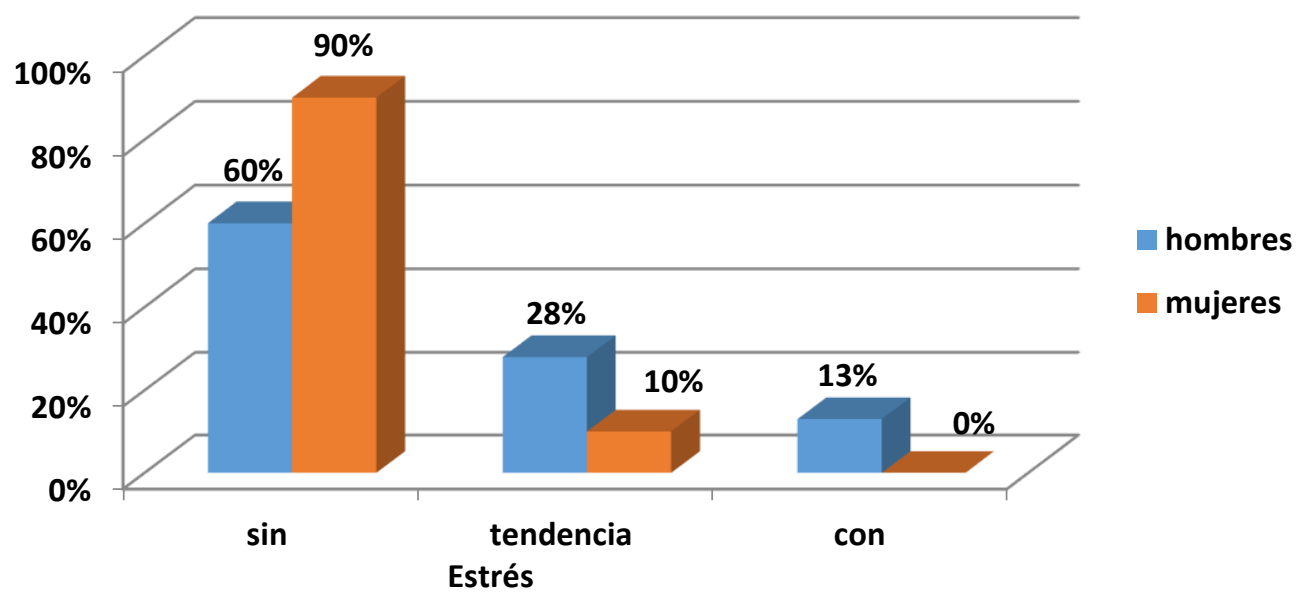

Figura 1. Nivel de estrés en trabajadores de Ingenio la Gloria, Veracruz. 
Con respecto a los hábitos de salud son conductas específicas que cuando se practican de manera regular, conduce tanto al bienestar físico como psicológico. La escala se compone por reactivos que se agrupan en cuatro áreas principales, cada una de las cuales constituye una subescala: Ejercicio, descanso. Sueño alimentación-nutrición y prevención. Ver Figura 2

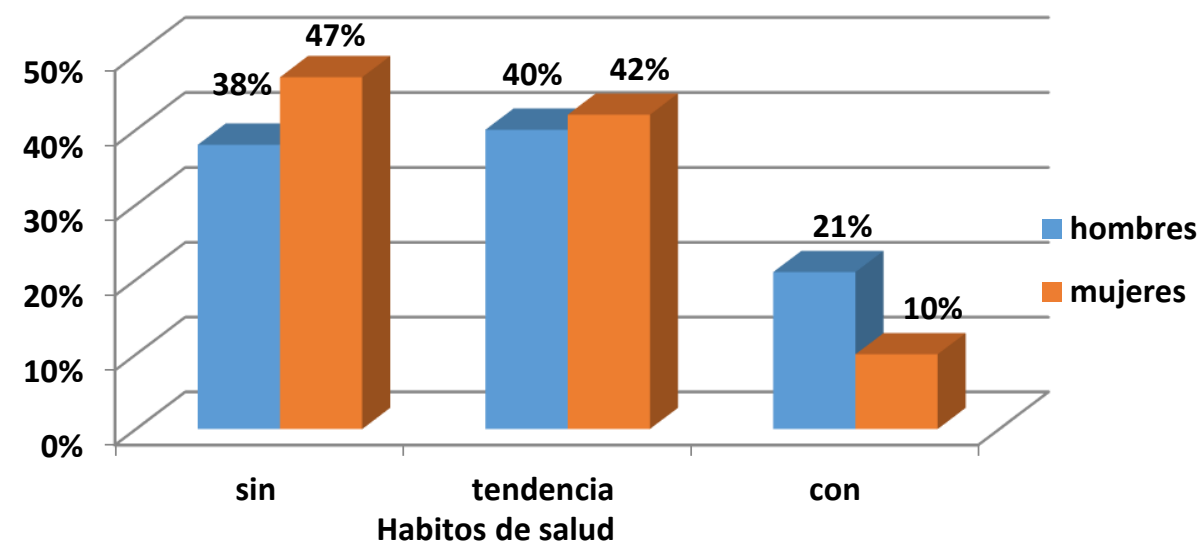

Figura 2. Hábitos de salud en trabajadores de Ingenio la Gloria, Veracruz

En cuanto a los factores que integran los hábitos de salud tenemos la siguiente descripción:

- Ejercicio.- En esta subescalas se mide el nivel y la frecuencia del ejercicio que el individuo practica de manera regular para mejor el tono muscular y el sistema cardiovascular,

- Descanso-sueño: Aquí se explora la frecuencia con la que el individuo obtiene descanso adecuado, sueño y relajamiento.

- Alimentación-nutrición.-En estos reactivos se valora si la persona tiene una práctica general comer de manera balanceada y nutritiva.

- Prevención.- Aquí se mide la frecuencia con la que el individuo pone en práctica estrategias preventivas de salud e higiene. Además, se explora el uso de sustancias que están asociadas con una incidencia alta de problemas médicos. Ver figura 2 


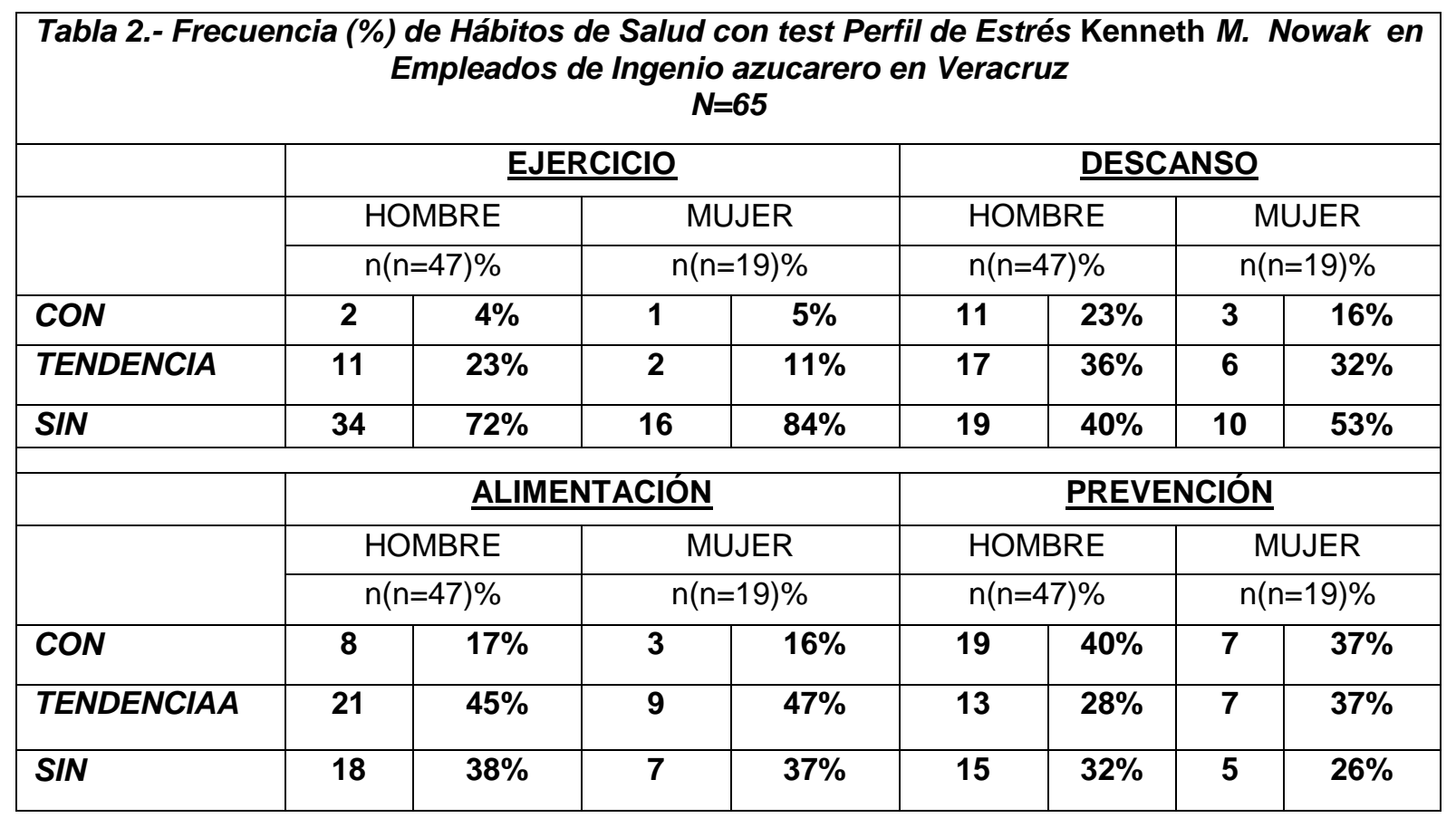

Fuente: Propia

a) En cuanto a la Conducta tipo A.- Mide el rango completo de las respuestas Tipo A expresadas, inclusive la ira internalizada, la ira expresada, premura de tiempo, rapidez laboral, impaciencia, involucramiento en el trabajo, búsqueda de mejoría, conducción ruda de vehículos y conductas competitivas. Ver. figura 4

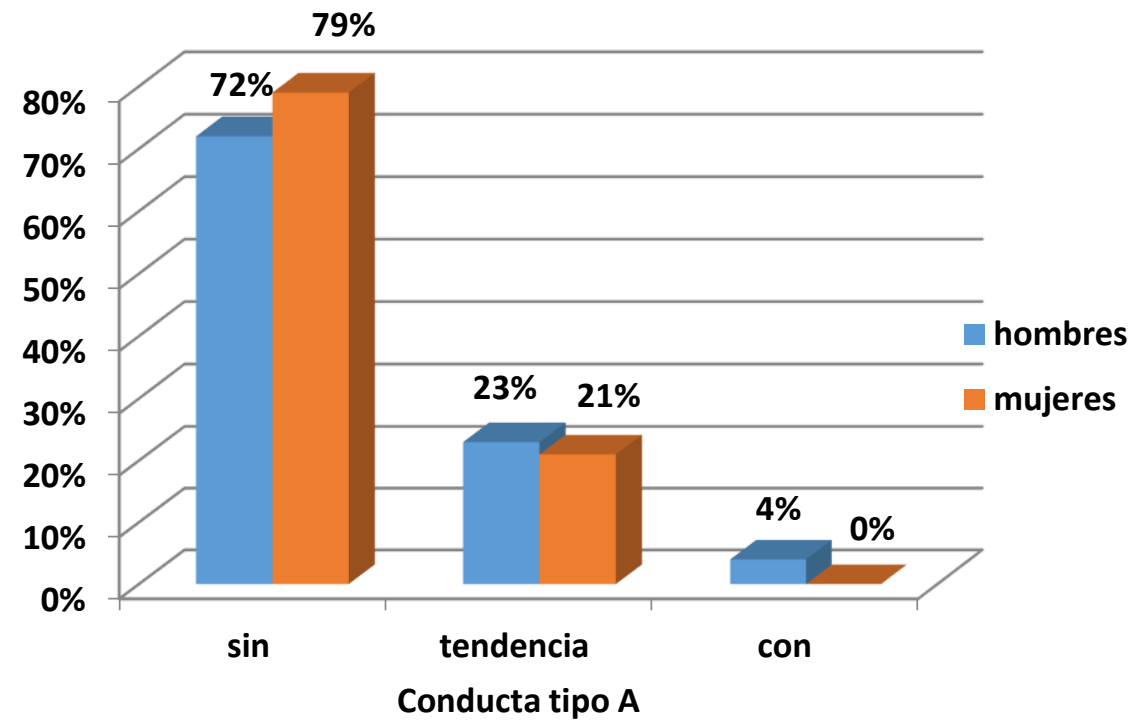

Figura 4. Conducta tipo A en trabajadores de Ingenio la Gloria, Veracruz. 
A) Fuerzas cognitiva.- Explora las atribuciones, actitudes y creencias que un individuo tiene acerca de la vida y el trabajo. Ver Figura 5

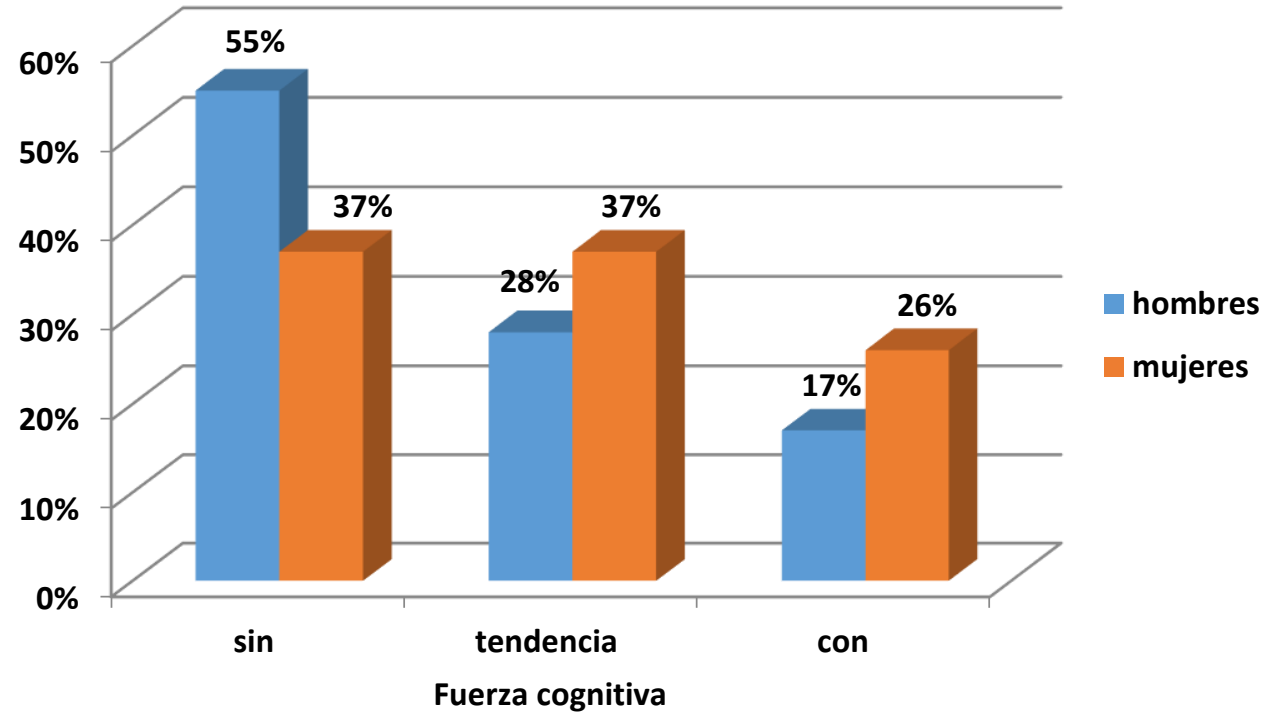

Figura 5. Fuerza cognitiva en trabajadores de Ingenio la Gloria, Veracruz.

A) Red de apoyo social.- Esta escala proporciona una medición directa del grado en el que el individuo siente que hay gente con la que puede contar en todo momento para obtener apoyo emocional, consejo, información, amor incondicional y ayuda, además de que tan satisfecho se siente con dicho apoyo. Ver figura 6 


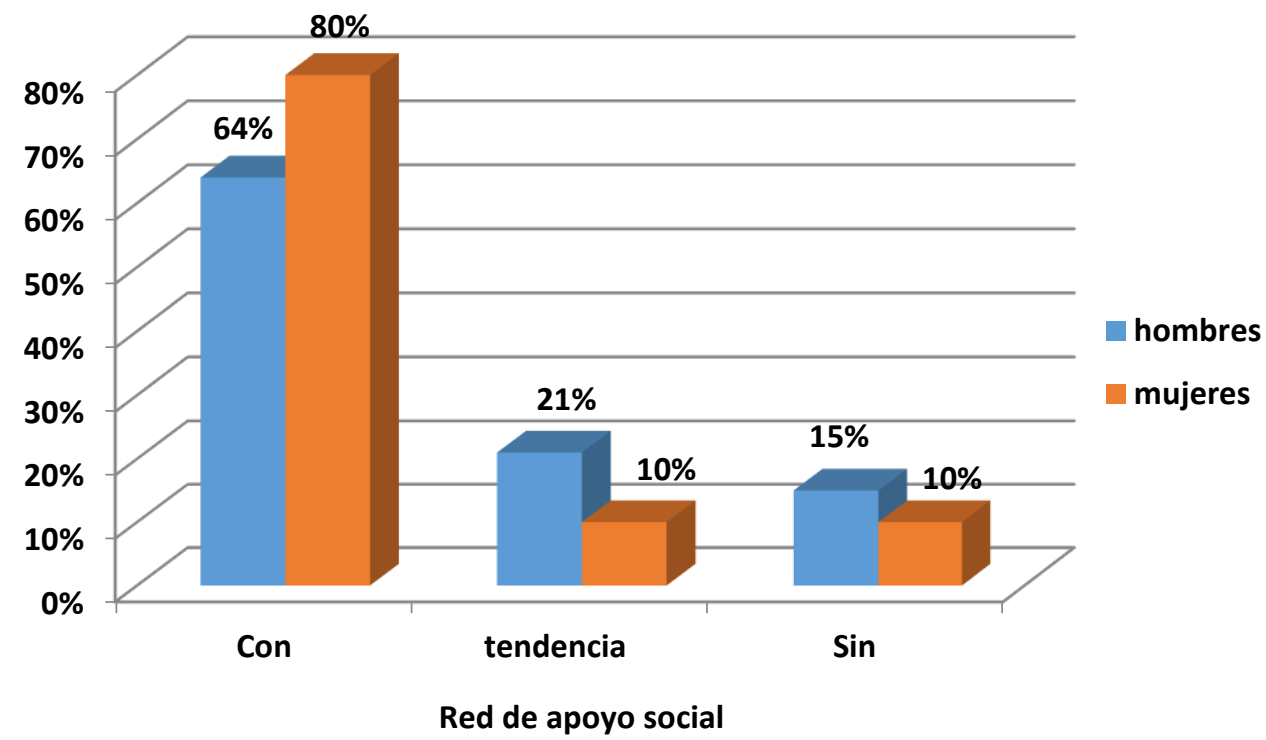

Figura 6 Red de Apoyo en empleado de Ingenio azucarero en el estado de Veracruz

Con respecto al Conglomerado de reactivos ARC/ uso del alcohol, drogas recreativas y cigarrillos. Ver Figura 7

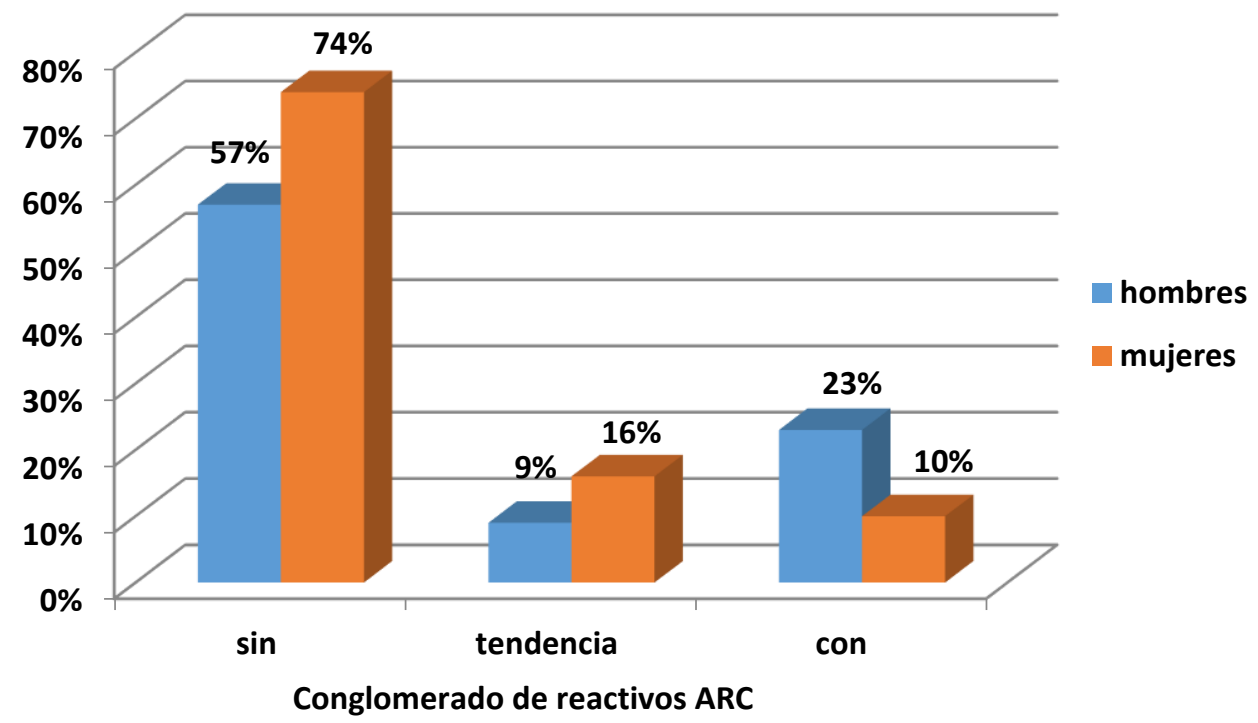

Figura 7. Conglomerado de reactivos ARC en trabajadores de Ingenio la Gloria, Veracruz. 
Con respecto a los estilos de afrontamiento--Valora cuatro estrategias de afrontamiento que tienen una base conceptual diferente: Valoración positiva, Valoración negativa, Minimización de la amenaza y Concentración en el problema

- Valoración positiva.- Explora el uso de comentarios de apoyo y motivación autodirigido para minimizar la percepción del estrés.

- Valoración negativa.-Mide la tendencia a aproximarse a las situaciones desafiantes desde esta perspectiva.

- Minimización de la amenaza.- Explora la tendencia del individuo de afrontar los estresores mediante la mitigación de su significación o no extenderse en ellos.

- Valoración positiva.- Explora el uso de comentarios de apoyo y motivación auto dirigido para minimizar la percepción del estrés.

- Valoración negativa.-Mide la tendencia a aproximarse a las situaciones Desafiantes desde esta perspectiva.

- Minimización de la amenaza.- Explora la tendencia del individuo de afrontar los estresores mediante la mitigación de su significación o no extenderse en ellos.

- Concentración en el problema.-En esta escala se indaga sobre la inclinación del sujeto a hacer intentos activos de cambiar su conducta o los estresores ambientales. Ver Tabla 3 


\begin{tabular}{|c|c|c|c|c|c|c|c|c|}
\hline Tabla 3.- Fre & & e Téc। & & $\begin{array}{c}\text { amiel } \\
\text { el Ing } \\
N=\end{array}$ & tes & e Es & & \\
\hline & & ALORA & $\mathbf{P}$ & & & ORAC & A & \\
\hline & & BRE & & ER & & & & \\
\hline & & .7)\% & & $9) \%$ & & & & 9)\% $\%$ \\
\hline CON & 22 & $47 \%$ & 7 & $37 \%$ & 6 & $13 \%$ & 0 & $0 \%$ \\
\hline TENDENCIA & 16 & $34 \%$ & 7 & $37 \%$ & 13 & $28 \%$ & 1 & $5 \%$ \\
\hline SIN & 9 & $19 \%$ & 5 & $23 \%$ & 28 & $60 \%$ & 18 & $95 \%$ \\
\hline & & IZACIC & $\mathrm{L} f$ & NAZA & $\underline{\mathrm{CO}}$ & RACIO & & LEMA \\
\hline & & BRE & & & & & & \\
\hline & & 7)\% & & $9) \%$ & & & & $9) \%$ \\
\hline CON & 23 & $49 \%$ & 8 & $42 \%$ & 14 & $30 \%$ & 5 & $26 \%$ \\
\hline TENDENCIA & 7 & $15 \%$ & 4 & $21 \%$ & 15 & $32 \%$ & 7 & $37 \%$ \\
\hline SIN & 17 & $36 \%$ & 7 & $37 \%$ & 22 & $47 \%$ & 7 & $37 \%$ \\
\hline
\end{tabular}

Fuente: Propia

En cuanto al Bienestar psicológico.- Valora la experiencia global del individuo de satisfacción y ecuanimidad psicológica durante los últimos tres meses. Ver Figura 8

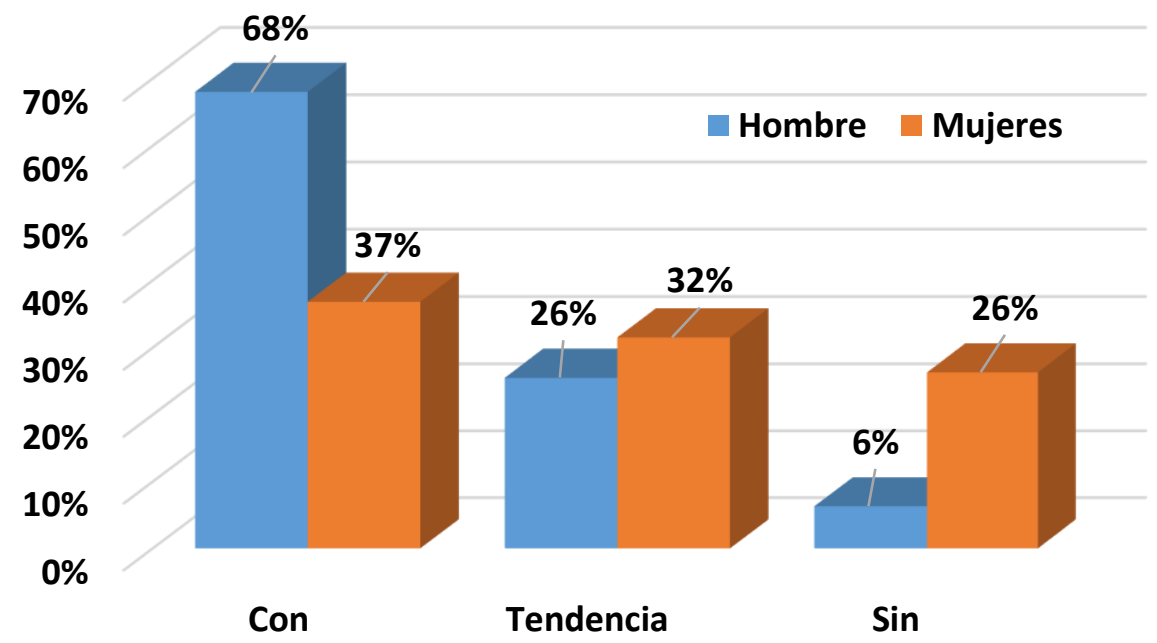

Bienestar Psicológicco

Figura 8. Nivel de Bienestar Psicológico en trabajadores de Ingenio la Gloria, Veracruz. 


\section{Conclusiones}

Considerando y dando respuesta al objetivo general en cual fue identificar el nivel de estrés, y técnicas de afrontamientos de empleados de diferentes departamentos de un Ingenio azucarero del Estado de Veracruz. Con el test "Perfil de Estrés" se realizan las siguientes conclusiones por cada escala de evaluación.

Con una distribución del $100 \%$ de acuerdo al sexo de los empleados tenemos que:

Estrés*: Tenemos que solo el 13\% de los hombres presentaron la mujeres no presentaron, sin embargo el $28 \%$ de los hombres y el $10 \%$ de las mujeres manifestaron una tendencia a presentarlo y sin estrés se encontró el $60 \%$ en hombres y $90 \%$ en mujeres. En una investigación realizada por Boza Ramos (2014) evaluó el estrés con el Perfil de estrés de Kenneth M. Nowarck en el personal del Grupo Pascual Boing de la ciudad de Veracruz, los resultados fueron: un $8 \%$ del personal presentó estrés, un 33\% tendencia y un 59\% sin estrés.

Hábitos de Salud: sin hábitos de salud se tuvo el 38\% en hombre y $47 \%$ en mujeres quiere decir que reflejan un riesgo para la salud. Véase figura 3, el $40 \%$ de hombres y $42 \%$ en mujeres presentan moderados hábitos de salud, quiere decir que practican conductas orientadas al mantenimiento de la salud de manera regular y finalmente el 215 en hombre y $10 \%$ si presentan buenos hábitos de salud, significa que mantienen un estilo de vida que satisface los estándares generales aceptados para mantener la salud Véase figura 2.

En cuanto a los factores que integran los hábitos de salud tenemos la siguiente descripción:

Ejercicio: El 72\% de hombres y el 84\% de mujeres no realiza actividades físicas regularmente, las cuales ayudan a disminuir la tensión y a prevenir el padecimiento de enfermedades cardiovasculares, además de fortalecer las redes de apoyo social mediante los ejercicios en grupo, Apenas el $23 \%$ de hombres y el $11 \%$ de humanidades si realizan actividades físicas con una regularidad de dos a tres veces por semana durante 20 a 30 minutos. Y solo el $4 \%$ de hombre y el $5 \%$ de mujeres si practican el ejercicio físico. Véase Tabla 2 
Descanso/ Sueño: En esta sub escala arrojó un el $40 \%$ de hombres y el $53 \%$ de mujeres que no mantienen un horario regular para el descanso, el cual podría ser un riesgo para satisfacer las exigencias laborales, sociales y personales. Solo el $36 \%$ de hombre y el $32 \%$ en mujeres mantienen un horario regular para satisfacer esta necesidad. Véase Tabla I

$>$ Alimentación/Nutrición: El el 17\% de hombres y el 16\% de mujeres presentan hábitos alimenticios regulares, el $45 \%$ de hombres y el $47 \%$ de mujeres tienden a mantener hábitos dietéticos balanceados, mientras que el el 38\% de hombres y el $37 \%$ de mujeres manifiesta riesgos de salud en sus hábitos de alimentación. Véase Tabla I

> Prevención: El 40\% de hombres y el 37\% de mujeres ponen en práctica estrategias preventivas de salud e higiene, mientras que El el 32\% de hombres y el $26 \%$ de mujeres muestra vulnerabilidad ante o riesgo ante enfermedad.

$>$ Conducta tipo A*: que el $\mathrm{El}$ el $4 \%$ de hombres y el $0 \%$ de mujeres expresan conductas de tipo A con mayor frecuencia; posiblemente debido a su auto exigencia para mantener su nivel académico; lo que podría ser propicio para desarrollar diferentes enfermedades por lo que se considera un riesgo muy importante para la salud, específicamente en las enfermedades cardiacas. mientras que El el $23 \%$ de hombres y el $21 \%$ de mujeres son menos autoexigentes y competitivos, además se enojan con dificultad y se comportan de manera afectuosa y amable. finalmente El el $72 \%$ de hombres y el $79 \%$ de mujeres No presentan conducta tipo A. Véase figura 4

Fuerza cognitiva: Poco más del $17 \%$ de hombres y el $26 \%$ de mujeres mencionan tienen Fuerzas cognitiva experimentan niveles altos de compromiso y participación en su trabajo, familia y consigo mismos, estás personas consideran los cambios de vida y los riesgo como una oportunidad de crecimiento personal y emocional. Mientras que El el 28\% de hombres y el $37 \%$ de mujeres dicen que es moderada su fuerzas cognitiva y El el $55 \%$ de hombres y el $37 \%$ de mujeres dicen no tener fuerzas cognitiva es decir una sensación de pérdida de control respecto los 
acontecimientos, no sobre los aspectos de importancia en su vida, esto último representa un riesgo para la salud. Véase figura 5

Red de apoyo social: , Se tiene que en "Red de Apoyo Social" sin presencia con un porcentaje el $64 \%$ para los hombres y un $80 \%$ para mujeres, con tendencia a un $64 \%$ para los hombres y un $80 \%$ para mujeres, mencionan manifiestan sentimiento de abandono al no contar con apoyo emocional o ayuda por parte de amistades, pareja o familia, mientras que el $21 \%$ para hombres y un $10 \%$ de mujeres dicen que es moderad su red de apoyo y el $64 \%$ para los hombres y un $80 \%$ para mujeres, tienen un nivel alto de red apoyo es decir se muestran satisfechos al sentirse apoyados por las personas, esto puede ayudar a que experimenten seguridad en cada dimensión de su vida. Véase figura 6

> Conglomerado ACR*: el 57\% para los hombres y un 74\% para mujeres se mantienen fuera de riesgo frente al consumo de (Alcohol, drogas o tabaco), el 9\% para los hombres y un $16 \%$ para mujeres tienen un riesgo moderado y el $23 \%$ para los hombres y un $10 \%$ para mujeres manifiesta un riesgo a la salud por consumo. Ver figura 7

\section{En cuanto a las técnicas de afrontamiento}

Valorización positiva: El $47 \%$ para los hombres y un $37 \%$ para mujeres manifiestan un alto nivel en valoración positiva es decir los empleados del ingenio azucarero, emplean estrategias de afrontamiento concentrándose en aspectos positivos de las situaciones que se presentan ante sí como estresantes, esto supone una automotivación para minimizar la percepción del estrés. el 34\% para los hombres y un $37 \%$ para mujeres tienen utilizan de manera moderada la estrategia de fuerza positiva, finalmente el $19 \%$ para los hombres y un $23 \%$ para mujeres señalan No tener o utilizar la técnica de afrontamiento de valor positiva. Véase Tabla 3

Valorización negativa*: En esta escala solo el 13\% para los hombres y un 0\% para mujeres consideran Manifestar un alto nivel en valoración negativa, el 28\% para los hombres y un 5\% para mujeres tienen utilizan de manera moderada la estrategia de valoración negativa, se concentran mayormente en los peores aspectos o consecuencias de una situación, el $60 \%$ para los hombres y un 95\% para mujeres 
señalan No tener o utilizar la técnica de afrontamiento de valoración negativa. Véase Tabla 3

- Minimización de la amenaza: Esta escala el 49\% para los hombres y un $42 \%$ para mujeres consideran utilizar la estrategia de minimización de la amenaza, el 15\% para los hombres y un $21 \%$ para mujeres tienden utilizan de manera moderada la estrategia de minimización de la amenaza. Esta evitación hacia las situaciones problemáticas, podría ser útil para el individuo en casos dónde no se ha manifestado algún tipo de problemáticas objetivas en su salud, es decir, en los casos de padecimientos crónicos esta estrategia podría conducir a un deterioro en la salud y conducir a la muerte. finalmente el 36\% para los hombres y un $37 \%$ para mujeres señalan No tener o utilizar la técnica de afrontamiento de minimización de la amenaza. Véase Tabla 3

> Concentración en el problema: el 30\% para los hombres y un 26\% para mujeres consideran utilizar la estrategia de concentración del problema, el 32\% para los hombres y un $37 \%$ para mujeres tienden a utilizan de manera moderada la estrategia de concentración del problema es decir muestran una inclinación a desarrollar intentos de cambios en su manera de actuar, desarrollando planes específicos de acción para dar solución a un conflicto analizando su propia experiencia o la de los demás para afrontar la situación estresante. , finalmente el 47\% para los hombres y un $37 \%$ para mujeres señalan No tener o utilizar la técnica de afrontamiento de concentración del problema. Véase Tabla 3

Bienestar Psicológico: se tiene que el 68\% para los hombres y un $37 \%$ para mujeres expresan satisfacción general y disfrutan de su vida, considerándose llenos de felicidad. Solo el $26 \%$ para los hombres y un $32 \%$ para mujeres dicen que es moderad Bienestar psicológico y el $6 \%$ para los hombres y un $26 \%$ para mujeres solo fue no tienen moderad Bienestar psicológico experimentan falta generalizada de bienestar en su vida, esto manifiesta un riesgo hacia su salud. . Véase figura 8

Sugerencias. Aprender a decir "no", no ser perfeccionista, aceptar lo inevitable, reconsiderar los "debería..." de su vida, tomar conciencia de que no puede cambiar a los demás, sólo a usted mismo, Seleccionar la información para evitar saturarse,, no magnificar ni subestimar los problemas, aceptar que no es posible tener todo lo que se desea, aprender 
a identificar lo realmente importante, saber perdonarse a sí mismo y a los demás, ver las situaciones críticas como una oportunidad. Considerar antes de salir a trabajar la planeación de su tiempo, tomar en cuenta la lista de tareas que ha programado realizar, Estando en su trabajo, tomar en cuenta la planeación de sus tareas asumiendo que está poniendo todo de su parte y si hay alguna que no alcanzo a realizar aceptar que no fue por negligencia, dese un tiempo de descanso, distrayéndose con alguna actividad que le agrade, consideré importante tener organizado su lugar de trabajo. Al finalizar su día de trabajo realice actividades que lo hagan sentir sano y feliz. 


\section{Bibliografía}

Furnham, Adrián (2009) Psicología Organizacional: el comportamiento del individuo en las organizaciones. México. Editorial Alfa omega.

Hernández, S.R, Fernández C.F, Baptista L.P. (2010) Metodología de la investigación. México. Editorial Mc Graw Hill Quinta edición.

Ivancevich, John M (1989) Estrés y trabajo: una perspectiva gerencial 2da edición. México: editorial trillas.

José María Peiró Silla (1999) Desencadenantes del estrés laboral. España. Ediciones Pirámide.

Lacan. J (1992) El seminario de Jaques Lacan. Libro 16 "De un otro al otro". Barcelona España. Editorial Paidós Ibérica, S.A.

Organización Internacional del Trabajo (2015) "Día Mundial de la Seguridad y Salud en el Trabajo - 28 de abril" Recuperado de http://www.ilo.org/global/topics/safety-andhealth-at-work/lang--en/index.htm

Robbins. S, Timothy A. (2005) Comportamiento Organizacional. México. Editorial Pearson, $13^{\circ}$ edición.

Sánchez, C., y Martínez, S. (2014). "Condiciones de trabajo de docentes universitarios, satisfacción, exigencias laborales y daños a la salud”, en: Salud de los trabajadores, Vol. 22(1), enero-junio, México, pp. 19-28.

Santiago, J; Tornay, F; Gómez, E. (1999) Procesos Psicológicos Básicos. España. Editorial Mc Graw Hill.

Stephen W, Cooper L (2004) Manejo del estrés en el trabajo. Plan de acción detallado para profesionales. México. Editorial: Manual Moderno.

Vygotsky, L. S. (1978). Pensamiento y lenguaje. Madrid. Editorial: Paidós.

Troch, A. (1982) El estrés y la personalidad. Barcelona. Editorial: Herder. 